\title{
Research on Factors Affecting Laser Welding Quality of Automobile Body
}

\author{
Zhang Jie, Ye Lan, Zou Jinhong \\ Jiangxi Engineering Laboratory of Digital Manufacturing for Automobile Parts, Nanchang institute of \\ science and technology, Nanchang 330108, P. R. China
}

Keywords: laser welding, automobile body, influencing factor

\begin{abstract}
Laser welding technology is featured by high speed, high efficiency and high quality, as it is widely used in automobile body welding. Laser welding technology can improve the quality of automotive body, reduce production costs of enterprises, for the development of new automotive products to provide technical support and quality assurance. This paper introduces the laser welding technology and its application in automobile body welding, and studies the influencing factors of the body laser welding quality. It is hoped that it can provide some reference for laser body welding technology.
\end{abstract}

\section{Introduction}

At present, our country has become the largest automobile consumer in the world. The automobile industry has become the pillar industry in our country and an important part of the national economy. The rapid development of the automobile industry has brought about such problems as energy consumption, environmental pollution and safety. At the same time, consumers are increasingly demanding on the quality and appearance of automobiles, prompting car developers to continually explore new materials and develop new technologies to meet market demand. Laser processing technology is the 21st century the most important optoelectronic technology, has been widely used in the automotive industry, embodied in the parts of the welding and cutting. The use of laser welding technology for the car body can improve the material strength, reduce the body quality, reduce production costs, bring significant benefits to the manufacturer.

\section{Laser welding technology}

Laser processing technology is one of the most important inventions since the 20th century, including laser welding, laser cutting, laser hardening and so on. Laser welding is a kind of fusion welding, the laser as an energy source, its impact on the metal surface, the laser and the metal reaction produces a lot of heat to melt the metal, so as to achieve the purpose of welding.

According to the actual work of the power density can be divided into heat conduction welding laser welding and deep penetration welding. Thermal conductivity welding laser spot power density of less than $105 \mathrm{~W} / \mathrm{cm} 2$. The part of the laser that strikes the welding surface is bounced back and the part absorbed by the weldment is transformed into heat that melts the material. Heat conduction welding power, low energy conversion rate, melting zone shallow, slow work, mainly for thin $(<1 \mathrm{~mm})$, small parts processing. Laser deep penetration welding power density is greater than $105 \mathrm{~W} / \mathrm{cm} 2$. When the laser beam is irradiated to the surface of the workpiece, a large amount of thermal energy is generated so that the metal is rapidly vaporized into steam. The metal vapor is separated from the surface of the workpiece to generate a certain reaction force, and the reaction force pushes the solution to form a pit. Subsequent laser irradiation continued in the pit, increasing the depth of the pit, the final formation of holes. When the laser stops irradiating, the liquid metal is reflowed to form a weld. Laser deep welding fast welding speed, high efficiency, used in the processing of large thick pieces.

In modern industrial production, laser welding has been widely used, laser welding compared with the traditional welding methods, and has the following advantages:

1) Welding speed, high efficiency. The use of high laser spot power density can be very fast 
welding speed (up to $20 \mathrm{~m} / \mathrm{min}$ ), and laser welding can achieve multiple simultaneous welding, high work efficiency.

2) High welding accuracy. Laser beam easy to control, focus, alignment is simple, and can improve the welding accuracy through optical instruments, can be applied to the welding of electronic components.

3) Weld high quality. Laser welding welding speed, work spot is small, the heat generated by a high degree of concentration. In the faster cooling rate weld joint refinement of the organization, the formation of weld aspect ratio, weldment deformation small.

4) High flexibility. Laser welding does not require a special environment, can work under air or vacuum conditions, easy to achieve long-distance or small and closely spaced parts welding.

5) Excited welding is non-contact welding, work will not wear the electrode, the formation of the weld uniform, high strength, will not affect the quality of the workpiece.

6) Solderable refractory metals and dissimilar metals. The laser beam can be focused on fine spots with high energy density, which can vaporize almost all materials, can be used for high melting point, high reflectivity metal welding, but also for different dissimilar metals welding.

7) Can be automated. Laser welding can be controlled by computer and welding robot for high flexibility and automation.

\section{Laser welding in automotive body applications}

The car body is an important part of the car, which includes the white body and accessory parts. Car body related to the safety of the vehicle, economy and maneuverability, while car personalization is also shown through the body design.

Automotive body welding process is an important part of the car production process; a reasonable advanced welding process can improve the car's productivity and reduce production costs. As early as the 1980s, the United States introduced laser processing technology into the automobile manufacturing industry. In 1996, Volkswagen began mass production of automotive bodywork using laser welding. Figure 1 shows the application of laser welding in the bodywork. Data show that laser welding saves more than $60 \%$ of the body's production molds and fixtures, shortens the production cycle by $50 \%$ and reduces the body weight by $20 \%$. While laser welding equipment is costly to place, it offers tremendous advantages over traditional welding techniques, significantly improving the quality of the bodywork and recovering investment costs within a few years.

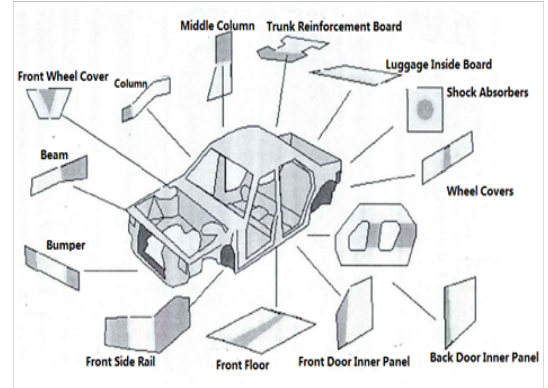

Figure 1. Laser welding in the body of the application

Figure 2 is a car body laser manufacturing process. Since the advent of laser welding, the automotive industry at home and abroad on the laser welding of a large number of metals. In recent decades, the theory and practice of laser welding technology has matured, and has yielded a lot of results. In addition, the laser welding market is fierce competition, prompting the laser welding equipment manufacturers to continuously improve product quality; the price has been declining, thereby promoting the further development of laser welding technology. According to the statistics, more than $50 \%$ of body parts of the automobile industry in the United States are manufactured by laser welding. Laser welding technology is also mostly used in automobile body production of well-known international automobile companies. Domestic automobile brands such as Chery, Geely, 
and The new models have laser welding technology applied.

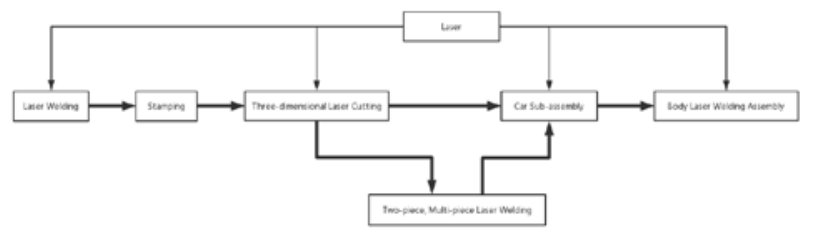

Figure 2. Automotive body laser manufacturing process

\section{Influencing factors of laser welding quality}

Automotive body laser welding process is complex, the operation is difficult, in the welding of weldment positioning accuracy and precision assembly requirements of the workpiece is very high, so the welding process parameters on the weld quality has a great impact. Automotive body laser welding quality factors are:

1) Material parameters, including weldment thermal physical properties, thickness, laser absorption and surface coating conditions.

2) Beam parameters, including beam quality, laser mode, focal length and focal spot size.

3) Process parameters, including laser power, welding speed, defocus and shielding gas.

4) Filler parameters, including the type of filler, feeding method, feeding angle and feeding speed.

5) Other parameters, including assembly accuracy of the workpiece, connector positioning accuracy and workpiece placement angle.

\subsection{Material parameters}

Material parameters determine whether laser welding technology can be used. The most important thermophysical properties in material parameters are the laser absorptance and the surface coating, which determine the solderability of the part. Such as the use of laser welding aluminum steel dissimilar materials, due to the thermal physical properties of aluminum and steel vary greatly, must be special welding process measures to ensure the performance of welding. In addition, aluminum, copper and their alloys have a high reflectivity of laser light at room temperature. The surface oxide layer needs to be removed before soldering. When using laser to weld a workpiece with unequal thickness, the workpiece is usually placed horizontally so that the upper surface will have a wrong edge due to the difference in thickness, resulting in uneven laser energy distribution on the two workpieces. The focus position can be adjusted to the upper surface of the weld, the laser beams relative to the normal surface of the workpiece tilted at an angle to the sheet direction, the laser energy distribution.

\subsection{Beam parameters}

The beam parameters include the laser type, beam quality, laser mode, focal length, focal spot size, etc. These parameters are determined by the laser welding equipment and cannot be changed during the welding process. Laser welding technology used in the main types of laser CO2 laser, Dd: YAG laser, fiber laser and Disc laser. These lasers have their own advantages and disadvantages, as shown in Table 1.

Table 1. The advantages and disadvantages of different types of laser comparison

\begin{tabular}{ccccc}
\hline & $\mathrm{CO}_{2}$ laser & Dd:YAG laser & Fiber laser & Disc laser \\
\hline Electro-optical conversion efficiency & $10 \%$ & $3 \%$ & $30 \%$ & $15 \%$ \\
Maximum output power & $20 \mathrm{~kW}$ & $5 \mathrm{~kW}$ & $50 \mathrm{~kW}$ & $8 \mathrm{~kW}$ \\
BPP & 6 & 25 & $<2.5$ & 8 \\
Pump life / hour & 8000 & 1000 & $>20000$ & 10000 \\
stability & good & bad & good & good \\
Maintenance and operating costs / hour & $\$ 20$ & $\$ 35$ & $\$ 2$ & $\$ 8$ \\
price & Low & Lower & higher & higher \\
\hline
\end{tabular}


Although the beam parameters cannot be changed during the welding process, they have a great influence on the quality of the weldment, especially the focal spot size. Therefore, the focal spot size has a great influence on the quality of the laser welding. When using fiber laser welding, to ensure the quality of the beam under the premise of stability, you can focus through longer distances to get a larger size of the focal spot for higher quality weldment. However, increasing the focal spot size will reduce the power density, thus reducing the welding speed, which is the need to change the other process parameters to ensure efficiency.

\subsection{Process parameters}

In the case of other parameters have been identified, the adjustment of process parameters can further improve the quality of welding.

Laser welding is based on thermal effects and heat input is controlled by laser power. The distribution of laser energy is controlled by the process parameters such as welding speed, welding time and defocusing. Under normal circumstances the welding quality is very sensitive to the welding speed, too fast too slow will affect the welding quality. The ratio of laser power to welding speed can be defined as the welding line energy. In the case of a fixed laser power, reducing the welding speed will increase the line energy, increase the heat input of the welding process; to improve the welding speed is just the opposite, while too much welding speed will lead to splashing.

The amount of defocus is also an important parameter affecting the quality of laser welding, which together with the focal spot size determines the laser spot size. By defocus method to increase the spot size, and increase the laser power, in order to ensure a constant power density and improve the laser bridging capability. At the same time, the study also found that the use of defocusing process to reduce fiber laser welding process of splashing.

\subsection{Other parameters}

In the laser welding process, the welding joint positioning accuracy is very important. Currently in the process experiment, the guide is usually used to guide the joint positioning, this visual way will cause some error. In practical industrial applications, the joint positioning is controlled by the coordinates, this method has high positioning accuracy.

In the actual laser welding applications, the location of the weld is uncertain, so the workpiece placement angle is uncertain. The most widely used workpiece placement methods are: flat (corresponding to flat welding), stand put (corresponding vertical welding.) Different parts placed in the metal, the direction of gravity by the molten metal is also different, the formation of the weld shape different.

The assembly accuracy of the workpiece is a prerequisite for laser welding. Only after the assembly accuracy of the workpiece reaches the standard, can the laser welding is used. Workpiece assembly accuracy defects include butt gaps, butt joints and oblique butt gaps, as shown in Figure 3 . When the butting gap is too large, laser welding cannot be used for machining. Different materials on the laser absorption rate is different, the allowable docking gap is also different. The wrong side of the butt welding has little effect on the welding process, but it has a great influence on the weld forming. Oblique butt gap is a kind of defect in the practical application of laser welding.

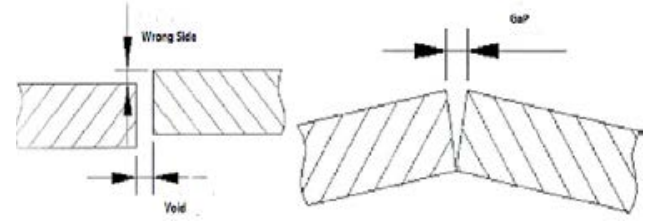

Figure 3. Workpiece assembly accuracy defects

\section{Conclusions}

Laser welding technology is to promote the development of the automotive industry; one of the advanced processing technology, in the automotive production has been more and more applications. 
Laser welding technology used in automotive body welding, can significantly improve the strength and height of the body; reduce the weight of the body, saving production costs. This paper mainly studies the factors affecting the quality of laser welding, and hopes to have a certain reference value for the application of welding technology in the automobile body.

\section{Acknowledgments}

This work was supported by the Nanchang Institute of Science and Technology research project for Jiangxi Engineering Laboratory of Digital Manufacturing for Automobile Parts under Grant No. SZZX-17-24.

\section{References}

[1] Zhang Yan. Laser welding in the body of the car on the status quo [J]. Welding machine, 2016,46 (03): $122 \sim 126$.

[2] Xu Xinpei, Luo Haiguo, Tang high school and so on. Automotive white body manufacturing advanced technology research - welding technology [J]. Equipment Manufacturing Technology, 2015 (02): 145 147.

[3] Dong Shaobin. Application of laser welding plate technology in automobile body manufacture [J]. Automotive Technology and Materials, 2006, (5): 9 11. 